\title{
Ultrasonic Studies of Emulsion Stability in the Presence of Magnetic Nanoparticles
}

\author{
A. Józefczak and R. Wlazło \\ Institute of Acoustics, Faculty of Physics, Adam Mickiewicz University, Umultowska 85, 61-614 Poznań, Poland \\ Correspondence should be addressed to A. Józefczak; aras@amu.edu.pl
}

Received 8 September 2015; Accepted 25 October 2015

Academic Editor: Da-Ren Hang

Copyright ( 2015 A. Józefczak and R. Wlazło. This is an open access article distributed under the Creative Commons Attribution License, which permits unrestricted use, distribution, and reproduction in any medium, provided the original work is properly cited.

Pickering emulsions are made of solid particle-stabilized droplets suspended in an immiscible continuous liquid phase. A magnetic emulsion can be obtained using magnetic particles. Solid magnetic nanoparticles are adsorbed strongly at the oil-water interface and are able to stabilize emulsions of oil and water. In this work emulsions stabilized by magnetite nanoparticles were obtained using high-energy ultrasound waves and a cavitation mechanism and, next, their stability in time was tested by means of acoustic waves with a low energy, without affecting the structure. An acoustic study showed high stability in time of magnetic emulsions stabilized by magnetite particles. The study also showed a strong influence of an external magnetic field, which can lead to changes of the emulsion properties. It is possible to control Pickering emulsion stability with the help of an external stimulus-a magnetic field.

\section{Introduction}

Emulsions are a mixture of two immiscible liquids in which one of the phases is dispersed in the other phase as droplets [1]. This system is thermodynamically unstable so ordinary emulsions are stabilized by surfactants. These emulsions are widely used across many different industries, including plastics and polymers, road surfacing, pharmaceuticals, cosmetics, paints, detergents, and foods [2].

Another method of emulsion stabilization is the use of solid particles. Already in 1907, Pickering showed that the adsorption of solid particles on the surface of droplets can effectively stabilize an emulsion [3]. Solid (nano- or micro-) particles function in a similar way to surfactant molecules but certain differences in behavior are inevitable; for example, particles do not assemble into aggregates in the same way that surfactant molecules form micelles so solubilization phenomena are absent in the case of particles [4]. Solid particles exhibit partial wettability in each of the colloid phases, which is a requirement for the creation of an emulsion; the particles reduce the free energy of the fluid-fluid interface and become kinetically trapped. It is therefore possible to create an emulsion stabilized solely by solid particles, where each droplet is coated with a layer of particles $[4,5]$. The irreversible adsorption leads to extreme stability for certain emulsions and is in contrast to the behavior of surfactant molecules which are usually in a rapid dynamic equilibrium between the oil-water interface and the bulk phases. There is evidence that, in some systems, weak flocculation of particles improves emulsion stability [4]. That kind of an emulsion is called a Pickering emulsion. Particle-stabilized emulsions have a vast range of applications, notably in food processing, biomedicine, and petroleum industry [6].

Magnetic emulsions (material susceptible to external magnetic fields) have long been known but emulsions have been prepared using classical surfactants $[7,8]$. A stable $(\mathrm{o} / \mathrm{w})$ magnetic emulsion was obtained by the emulsification of a ferrofluid in an aqueous solution of surfactants having appropriate hydrophilic-lipophilic balance [7]. The properties of emulsions with ferrofluid droplets dispersed in water have been studied in several works. A ferrofluid emulsion subjected to a magnetic field exhibits complicated structural behavior: droplets aggregate to form threedimensional solid structures $[9,10]$. However, it is possible to obtain a stable magnetic emulsion without surfactant stabilization. Magnetic nanoparticles, for example, magnetite particles suspended in 


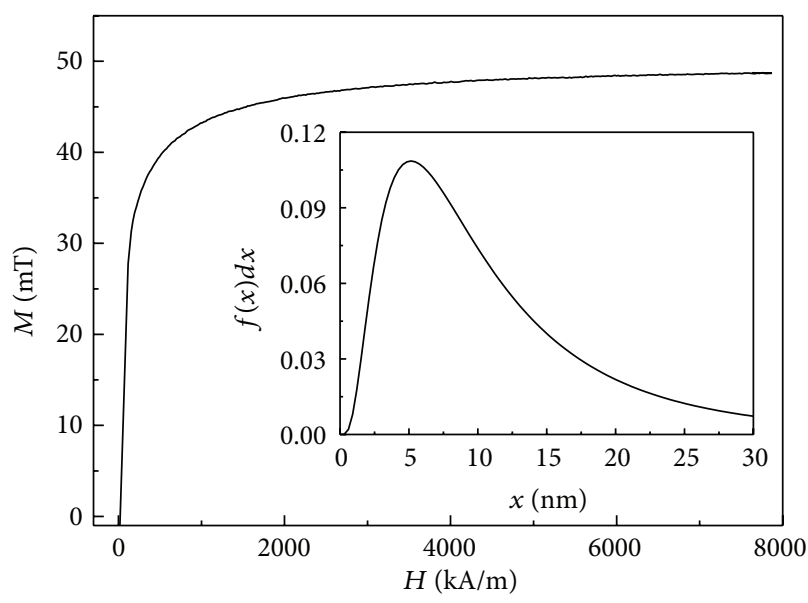

(a)

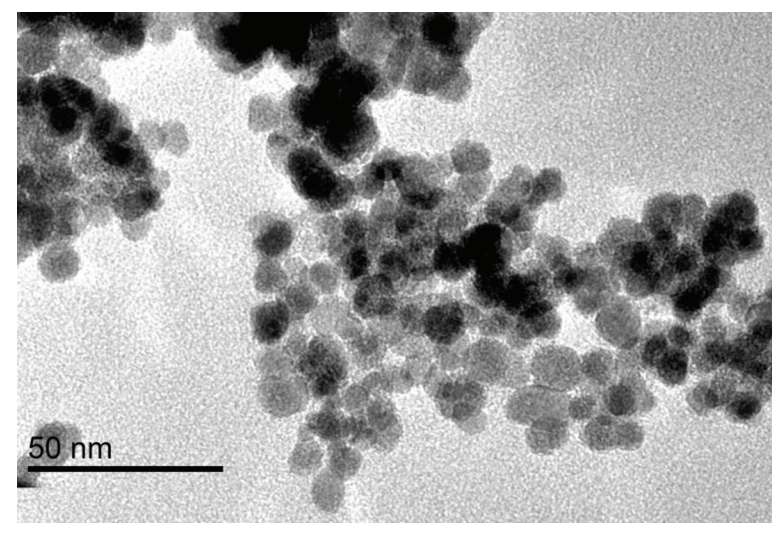

(b)

FIGURE 1: (a) Magnetization curves $M(H)$ for oil suspension of magnetic nanoparticles, obtained from SQUID and magnetite particle size distributions (inset). (b) Transmission electron microscope images of superparamagnetic nanoparticles prepared by coprecipitation method.

a ferrofluid, can be used to stabilize the Pickering emulsion [11-16]. This is how a magnetic Pickering emulsion is formed.

Pure particles or particles with a modified surface can be used in order to obtain the desired hydrophobic/hydrophilic properties. Many authors describe the use of magnetic particles modified by various substances, for example, silane, BSA, or a double layer of oleic acid [11, 13, 17]. In this paper oleic acid-modified magnetite nanoparticles were used to stabilize the transformer oil-in-water emulsion. The emulsions were obtained using high-energy ultrasound waves and a cavitation mechanism. In emulsification, ultrasound ranges used can be divided into high frequency/low energy ultrasounds and low frequency/high-energy power ultrasounds [18]. The low frequency ultrasounds use intensities higher than $1 \mathrm{~W} \cdot \mathrm{cm}^{-2}$ and frequencies to $1 \mathrm{MHz}$. The power available varies inversely with the frequency and only powerful ultrasounds $(16-100 \mathrm{kHz}$, and, to a lesser extent, $100 \mathrm{kHz}-1 \mathrm{MHz}$ ) interact with matter, producing physical and chemical changes [18]. The low energy ultrasounds have frequencies above $1 \mathrm{MHz}$ at intensities below $1 \mathrm{~W} \cdot \mathrm{cm}^{-2}$ and are usually used as a nondestructive and rapid technique for measuring the properties of materials, such as composition and structure. Using high frequency ultrasound to produce an emulsion is also possible [18]. That study of the effect of high frequency ultrasounds on the formation of oil emulsions droplets showed a decrease in the size oil of particle formed due to the deformation and breakage of oil droplets during the propagation of an acoustic wave [18].

After the preparation the stability of the emulsion in time was studied because emulsion stability is a critical parameter in a multitude of industries and applications. Many methods have been applied to the study of emulsion stability: optical methods (light scattering, photon correlation spectroscopy), dielectric constant or electric conductivity measurements, NMR, calorimetric, and ultrasonic measurements [1, 19]. Ordinary emulsions are often studied by means of ultrasonic waves with very low intensity, without the perturbation of the state of the material through which the waves travel.
In this work, an acoustic method was used for monitoring the stability of an emulsion stabilized by solid magnetic particles for a long time. Acoustic methods do not need any prior modifications of samples and are suitable for monitoring particle effects, flocculation, and coalescence processes, as well as phase transitions [19] which occur at a high concentration without destroying the system [2].

The effect of a magnetic field on the magnetic properties of magnetic Pickering emulsions was also investigated, using ultrasonic measurements. The study has shown the studied emulsions can be categorized as mixtures with magnetic fieldcontrollable properties.

\section{The Preparation and Characterization of a Magnetic Emulsion}

Superparamagnetic iron oxide nanoparticles (SPION) were first synthetized. The $\mathrm{Fe}_{3} \mathrm{O}_{4}$ nanoparticles were prepared by chemical precipitation using ferric and ferrous salts in an alkali medium. Freshly prepared magnetic nanoparticles were sterically stabilized by oleic acid $\mathrm{C}_{18} \mathrm{H}_{34} \mathrm{O}_{2}$ [20]. The thickness of the surfactant layer is $\delta=2 \mathrm{~nm}$ [21]. The purified magnetite nanoparticles were dispersed in inhibited mineral transformer oil with the density of $824 \mathrm{~kg} \cdot \mathrm{m}^{-3}$ and viscosity of $3.08 \mathrm{mPa} \cdot \mathrm{s}$ in $15^{\circ} \mathrm{C}$. Such a colloidal suspension of monodomain magnetic particles is called a ferrofluid. Figure 1 presents the SQUID measurements of the magnetization curve in room temperature. From the curve saturation magnetization, $M_{s}=0.0499 \mathrm{~T}$, the volume concentration of magnetite particles, $\phi=10 \%$, their size distribution, and mean diameter $\langle d\rangle=18 \mathrm{~nm}$ were determined [22]. The bulk magnetization of the prepared magnetite nanoparticles is equal to $M_{d}=334 \mathrm{kA} / \mathrm{m}$ [23]. Figure $1(\mathrm{~b})$ presents a transmission electron microscope image of superparamagnetic nanoparticles prepared by the coprecipitation method. The nanoparticle surface area to volume ratio in nanoscale is very large so surfactant (oleic acid) was used at the volume concentration of $17 \%$ in a ferrofluid [21]. 


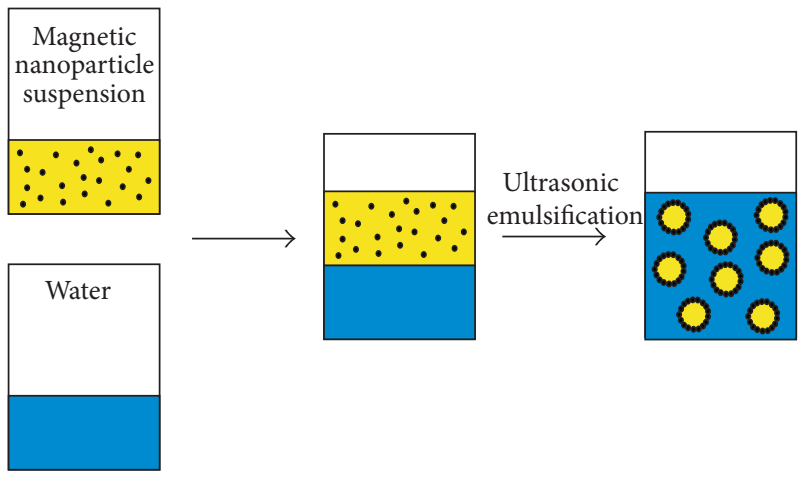

FIGURE 2: A schematic representation of the preparation method of a magnetic Pickering emulsion.

The oil suspension of nanoparticles was then added to water. Then an oil-in-water emulsion was prepared. Emulsions can be formed using a variety of devices, for example, a chemical stirrer, a vortex mixer, a homomixer, a hydroshear, a colloid mill, or an ultrasonic disruptor [1]. In this work, the emulsions were obtained using ultrasound waves and a cavitation mechanism. The emulsions were obtained by adding a magnetic nanoparticle oil suspension to water and sonicating the mixture. The apparatus for the emulsifying oil suspension of magnetic nanoparticles with water consists of a signal generator joined to a high frequency power amplifier model, AL-300-HF-A $(P=300 \mathrm{~W})$, which supplies the signal, through a transformer, to two piezoceramic rings (Pz-26 type produced by Ferroperm). Those rings are mechanically pressed with the help of a steel bolt as well as back (steel) and front (aluminum) vibrating masses. The front acoustic mass vibrates with an aluminum concentrator, the length of which corresponds to a half of the ultrasonic wave $(\lambda / 2 \cong$ $125 \mathrm{~mm}$ ) [24]. The tip of the concentrator is immersed in a liquid medium. The acoustic wave frequency at which the tip displacement has the maximum value was $18 \mathrm{kHz}$. A schematic representation of the preparation method of a magnetic emulsion is shown in Figure 2. The ultrasound emulsification process lasted 15 minutes. Emulsions with different oil/water volume ratios of $2: 1,1: 1$, and $1: 2$ were prepared.

The emulsion type was determined by a drop test $[25,26]$. A drop of the formed emulsion was added to water. The droplet dispersed in water so the emulsion type is o/w.

The Nikon's Eclipse E600POL optical microscope was used to observe the morphology of the emulsions. Figure 3 presents an optical micrograph of the prepared emulsion. It is clear that the dispersion of spherical droplets was achieved using ultrasound waves. In addition, it can be seen that the droplet sizes have high polydispersity.

\section{Ultrasonic Monitoring of the Stability Emulsion}

The stability of the prepared emulsions was assessed by measuring their acoustic parameters-the velocity and the attenuation of an ultrasonic wave in time. Acoustic

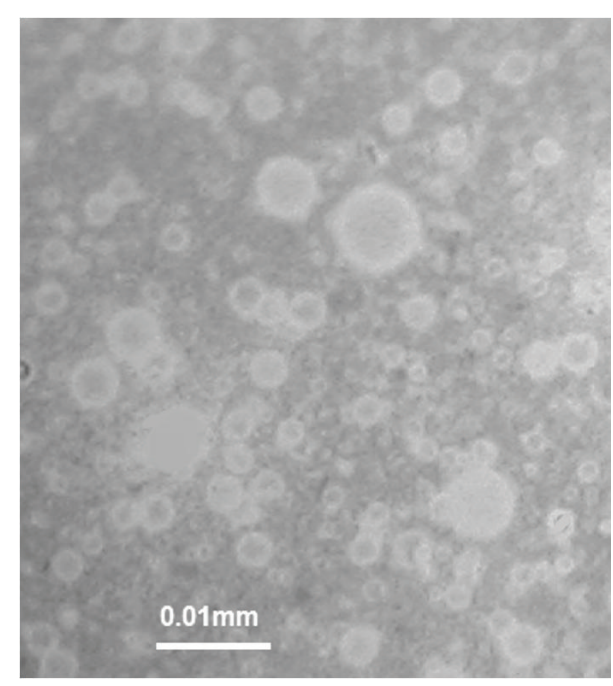

FIGURE 3: An optical micrograph of the emulsion with a $1: 1$ oil/water volume ratio.

measurements are suitable for characterizing not only stable emulsions but unstable ones as well. For instance, it is possible to monitor the changes in velocity and attenuation with time [19]. Sound velocity is more suitable for characterizing chemical reactions, whereas attenuation is sensitive to the processes and properties with the large space scale of nanometers and micrometers [19]. In this technique, an acoustic wave is passed through the sample that is being measured. When the wave travels through the sample, it loses energy; that is, it is attenuated. When the sample contains suspended droplets, the attenuation changes due to a variety of different scattering and absorption mechanisms [2]. Figure 4 shows a block diagram of the experimental set-up. The broadband transducer (Optel) with the center frequency of $4.5 \mathrm{MHz}$ was used in the ultrasonic measurements (in the inset of Figure 5(a) the frequency domain spectrum of the signal is presented). The piezoelectric transducer was driven by Optel Pulser/Receiver Card-01/100 which provided a unipolar spike pulse with the amplitude of $300 \mathrm{~V}$ and the rise time of $8 \mathrm{~ns}$. The pulse enters the studied emulsion and, after being reflected from the reflector, it is transformed by the transducer into voltage. Ultrasonic waves have a very low intensity so they are unlikely to perturb the macroscopic state of the material through which they travel. The received signal was sampled and recorded in the Optel Card. Figure 5(a) shows a typical time domain signal from the propagation through the colloid. Each emulsion was measured for 10 days at a constant temperature, $T=20^{\circ} \mathrm{C}$. Figure $5(\mathrm{~b})$ shows the changes of the signal recorded for 10 days. The changes of the signal amplitude and the time of flight were measured.

At the same time, the emulsions were placed in $4 \mathrm{~mL}$ vials and the stability of the emulsions to creaming and coalescence was assessed by observing the oil-water interface in the emulsion with time, in room temperature, and imaged using Canon photo camera. Photographs were taken at the same time as the acoustic measurements. 


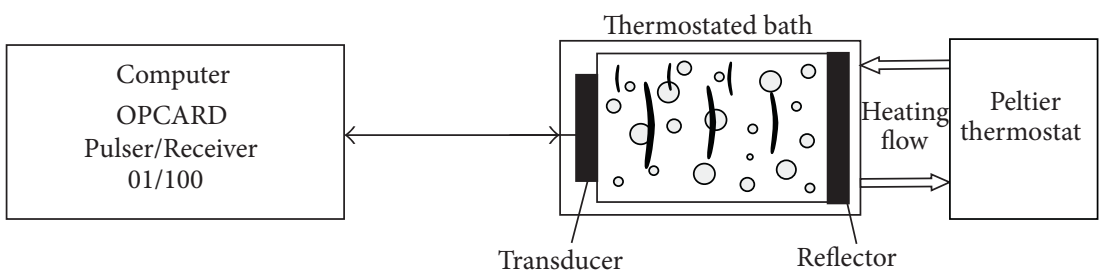

FIGURE 4: A block diagram of the experimental set-up for ultrasonic monitoring of emulsion stability.
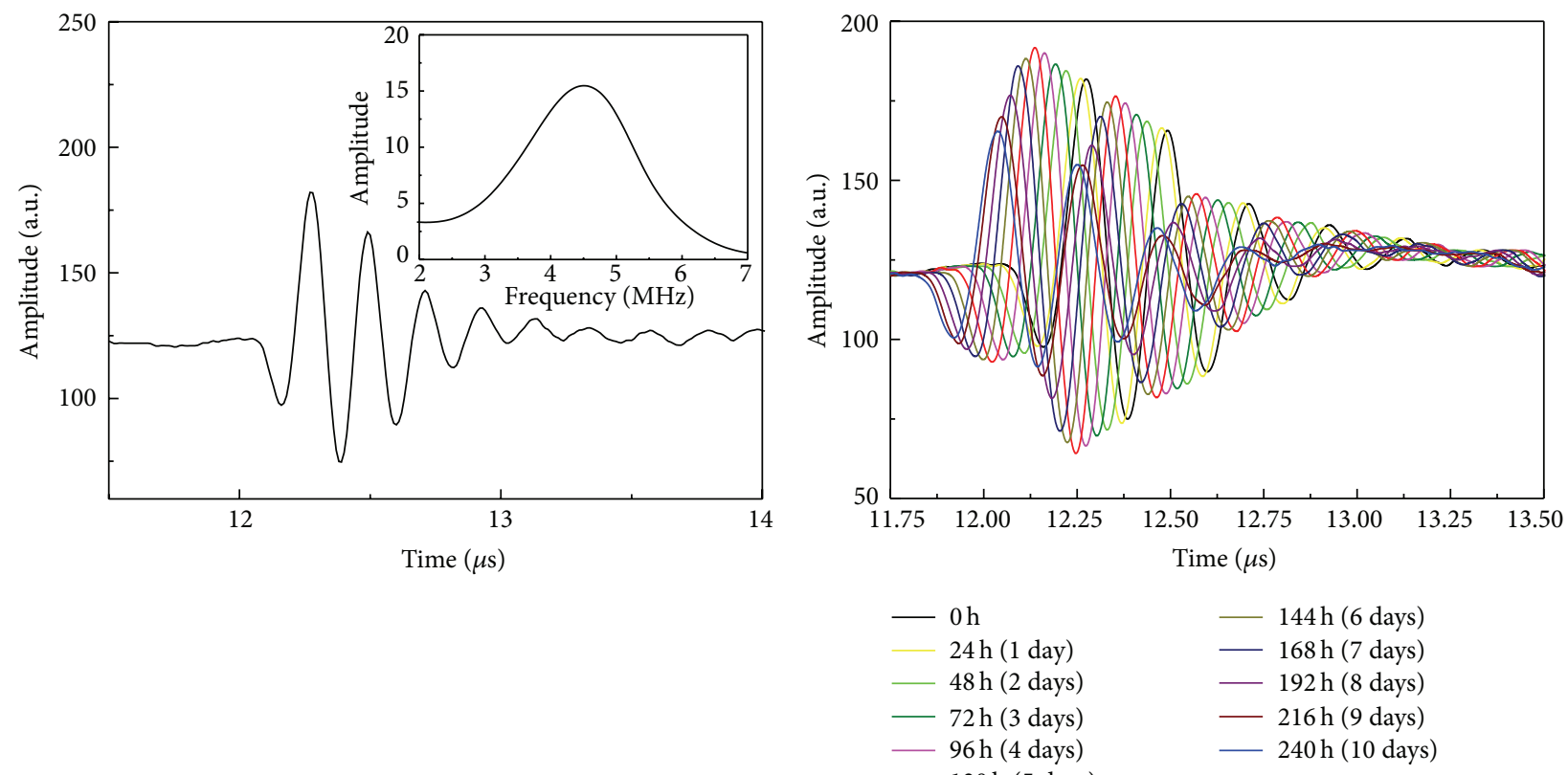

(a)

(b)

Figure 5: (a) The typical time domain received signal with the corresponding frequency domain spectrum (inset) and (b) the changes of signal recorded for 10 days.

The results of the stability monitoring of the prepared emulsions are presented in Figure 6. It shows the ultrasonic wave velocity and attenuation as a function of time for different oil/water volume ratios. Changes in the acoustic parameters were observed. The velocity of ultrasonic waves in emulsions decreases with the concentration of oil and magnetic nanoparticles. Magnetic fluids containing chemically synthesized magnetic nanoparticles behave similarly [21,22]. Only the suspension of magnetosomes (biogenic magnetic nanoparticles) behaves inversely [27]. Within 10 days very small changes in the propagation velocity of ultrasonic waves were observed which suggests good stability of the prepared emulsion. This is also confirmed by the measurements of the attenuation coefficient, which is better for characterizing the dispersed phase composition. Attenuation at low frequencies, below $10 \mathrm{MHz}$, can be used to study emulsion droplets [19]. The change in attenuation as a function of time at a single frequency $(4.5 \mathrm{MHz})$ is shown in Figure $6(\mathrm{~b})$. It is seen that the attenuation changes continuously over the course of the $240 \mathrm{~h}$ experiment. There are two effects occurring simultaneously. First, the attenuation decreases because of the creaming of the oil and the resultant decrease in the weight fraction of the oil phase which is still within the path of the sound beam. Second, in addition to that obvious creaming effect, the attenuation changes because of the flocculation and the evolution of the droplet size due to coalescence [19]. The highest absorption is observed in emulsion $1: 1$, which means that smaller droplets were created in that concentration. For a frequency about $5 \mathrm{MHz}$ in the range of 1 to several tens of microns the absorption of ultrasonic waves decreases with the size of droplets $[28,29]$. However, smaller droplets coalesce at a faster rate than the larger ones [19], so the greatest $\alpha$ changes in time were observed. The attenuation changes were small-up to $\Delta \alpha \approx 5 \mathrm{~m}^{-1}$-which indicates high stability as a function of time and very slow coalescence. The changes of attenuation caused by an external magnetic field (Figure 10) which lead to the destabilization of the emulsion are 10 times larger, $\Delta \alpha \approx 50 \mathrm{~m}^{-1}$.

Wettability has a great influence on emulsion stability. The wettability of the particles is changed by the addition of a surface active material [30]. Amphiphilic molecules which have a hydrophilic portion on one side and a hydrophobic part on the other side adsorb on the SPION surface. Particle wettability depends on surfactant concentration [26]. 


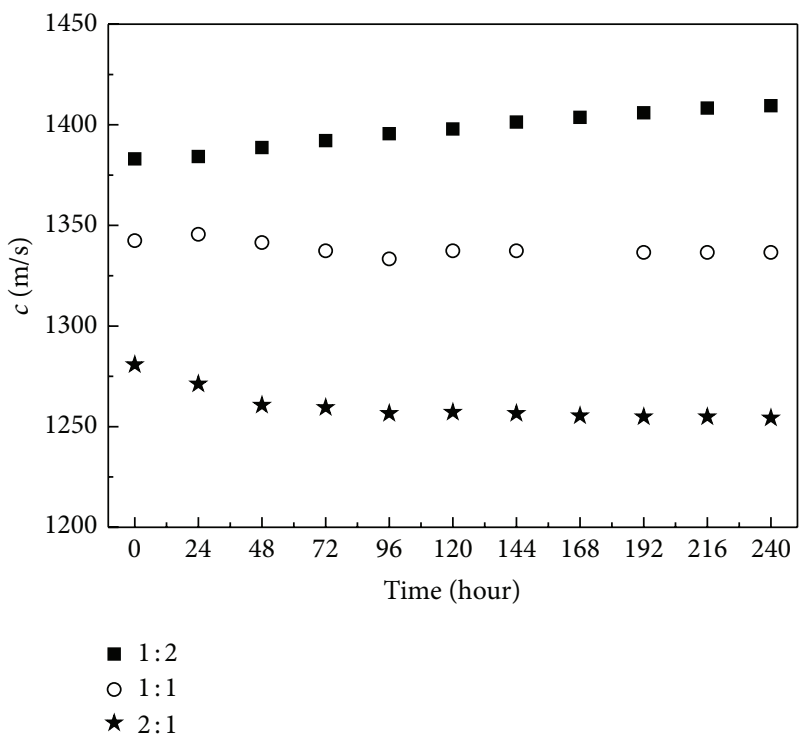

(a)

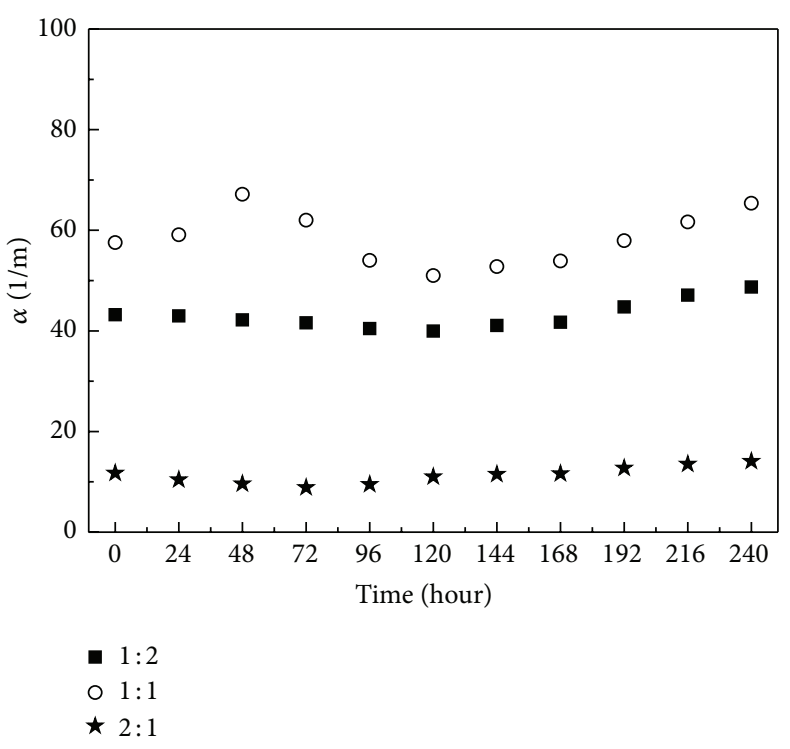

(b)

FIGURE 6: (a) The ultrasonic wave velocity and (b) attenuation, as a function of time for different oil/water volume ratios.

Without any surfactant, the surface of magnetite nanoparticles is naturally hydrophilic [31]. Positively charged surfactant head groups adsorb on the surface of the nanoparticles, exposing hydrophobic tails. For low surfactant concentrations, the surface is still mostly exposed, maintaining the hydrophilic character of the particles. Above a particular level of concentration, most of the particle surface presents hydrophobic tails, raising the particle hydrophobicity. Adding more surfactant, above the full monolayer coverage, leads to the formation of a bilayer in which the hydrophilic head groups are now exposed, and the particle hydrophilicity increases again [26]. The obtained stable emulsions indicate there is a suitable amount of a surfactant in the surface of the magnetic nanoparticles to provide appropriate hydrophilicity. That has been confirmed by this acoustic study and the observations of the images, shown in Figure 7. For the 1:2 and 2:1 emulsions the process of creaming can be observed-oil droplets accumulate on the surface-but the process is very slow. The photographs of the 1:1 emulsion show high stability-they do not present any changes-but acoustic measurements show slow changes on the nano- and microscale.

\section{Rheological Properties}

The measurements of viscoelastic properties were carried out using the rotation Digital Brookfield Rheometer DV II + in a cone-plate geometry. Figure 8 presents the shear stress $\tau$ as a function of the shear rate $\dot{\gamma}$ of the prepared emulsions. The measurements which were taken immediately after the preparation of the emulsion (fresh emulsion) versus the increasing shear rate in the range of $2.5-750 \mathrm{~s}^{-1}$ (series 1) show the influence of the oil and nanoparticles concentration on the viscosity of emulsions $(\eta=\tau / \dot{\gamma})$. Without a magnetic field, the emulsion particles exhibited typical non-Newtonian behavior [32]. The viscosity of the emulsion increases with

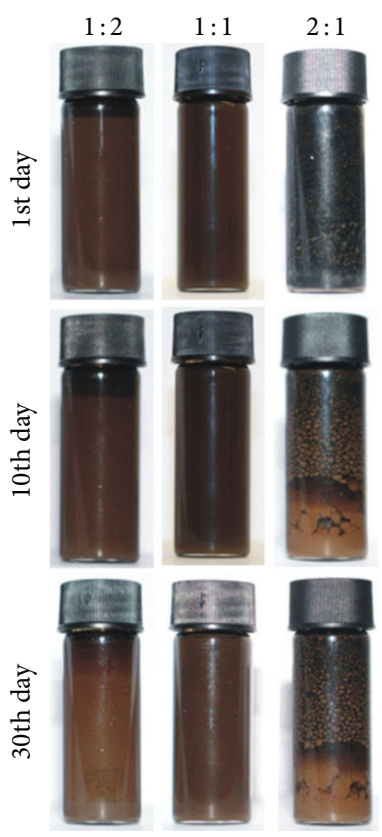

Figure 7: Photographs of fresh emulsions (1 day) and after 10 and 30 days.

the increase of the amount of nanoparticles. That behavior is typically observed in magnetic liquids [33].

A second series of rheological measurements was performed after that measurement cycle. The viscosity of the emulsions before and after the application of shear rate is different. It can be seen that the shear rate history influences the viscosity of the magnetic emulsions. Applying shear stress to an emulsion might cause two opposing effects: the coalescence of smaller droplets into larger ones or the disruption of larger droplets into smaller ones. [19]. The formation of 


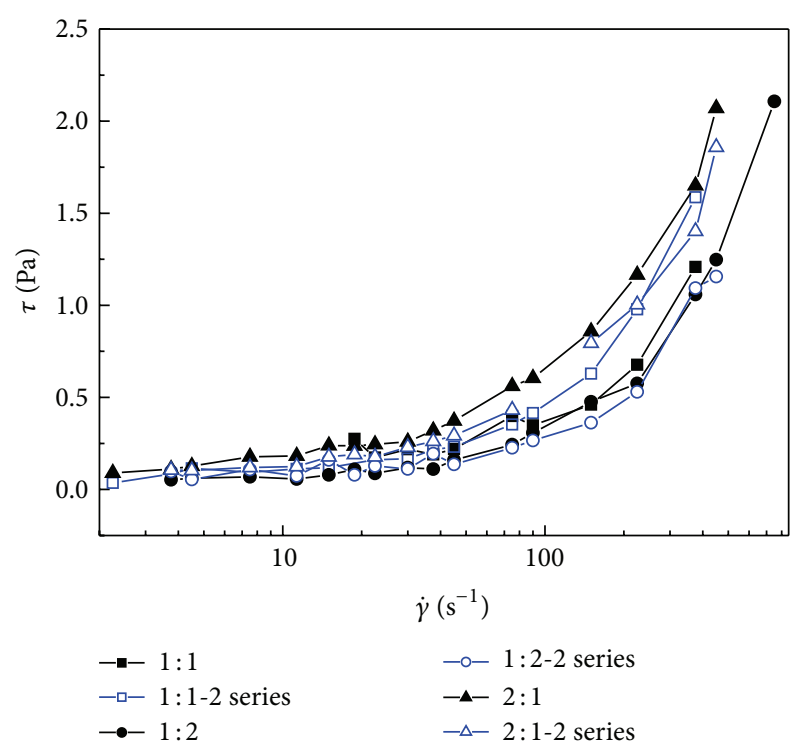

FIGURE 8: Shear stress versus shear rate flow curve of a fresh emulsion and of the emulsion after the application of the increasing shear rate in range $2.5-750 \mathrm{~s}^{-1}$.

droplet aggregates leads to the appearance of viscoelastic effects or other non-Newtonian features in an emulsion stabilized by solids particles. All emulsions exhibited shearthinning (viscosity decreasing with shear rate), probably as a result of the shear disrupting flocculated drops [4].

The propensity of emulsions to aggregate increases with the shear rate. Low shear rates create smooth, nonaggregating emulsions whilst progressively higher shear rates create increasingly flocculated networks of droplets. The characteristics of Pickering emulsions depend on the shear history of the sample. An increasing shear rate causes a decrease in the emulsion packing fraction [5]. At higher shear rates there will be a greater amount of interfacial area exposed, so a greater number of collisions between partially coated droplets will take place. Since it is those collisions that lead to particle bridging, the degree of aggregation increases when shear rate is increased [5]. The increased polydispersity of those emulsions is also the cause of changes of rheological properties. It is well known that the size distribution has a major effect on the suspension viscosity of particle suspensions-viscosity decreased when larger particles were added to a suspension of small particles [4]. The difference between series 1 and 2 is low. It indicates high stability of the prepared emulsions.

\section{The Influence of a Magnetic Field on the Emulsion}

For some application, long-term emulsion stability is critical. However, in other cases only temporary stability is desired. In these instances, additional physical and/or chemical disruption mechanisms must be introduced into the system to destabilize the emulsion. In an effort to simplify the demulsification process, Pickering emulsifiers should activate and deactivate in response to external stimuli (e.g., magnetic field). In many cases, the surface properties of solid particles undergo some physical or chemical transformation in response to environmental triggers that, in turn, alter the wettability of the particles. That enables the remote control of the emulsion characteristics [34]. The properties of an emulsion stabilized by magnetic nanoparticles are sensitive to an external magnetic field. Figure 9 illustrates the influence of a magnetic field on the behavior of the emulsions. A neodymium magnet was placed close to the wall of the vial containing the magnetite particle-stabilized emulsions. The photographs show that after the magnet has been put close to the emulsion the emulsion slowly deforms and collects toward the source of the field- $\mathrm{Fe}_{3} \mathrm{O}_{4}$ nanoparticles with droplets moving in a magnetic field. When a magnetic field is applied to a colloid, magnetic dipoles interact with one another, changing the structure of the colloids and causing structural transition [9]. The induced droplet magnetic moments grow with the field strength. Thus, the effective emulsion magnetic permeability increases. In a stronger magnetic field the droplets are highly elongated [35].

These induced structures also dramatically change the ultrasonic properties of the emulsions. After 10 days, two neodymium magnets were placed close to the measuring cell and changes in velocity and attenuation of ultrasonic waves were observed under the influence of a $150 \mathrm{mT}$ magnetic field. The measuring cell was placed between two neodymium magnets - the acoustic wave was propagated perpendicularly to the direction of the magnetic field $(k \perp H)$. Figure 10 presents the changes of the ultrasonic wave velocity and attenuation as a function of time after the application of a magnetic field and after switching off the field. The figure presents rapid changes in emulsions. The application of an external magnetic field influences both the velocity and attenuation of ultrasonic wave. The measurements showed that sound velocity is greater in the presence of a magnetic field because of the increased rigidity of the emulsions. According to the well-known Newton-Laplace equation $c=$ $\left(\rho \beta_{s}\right)^{-1 / 2}$, sound velocity $c$ is related to its density $\rho$ and the adiabatic compressibility $\beta_{s}$, so with increasing velocity the adiabatic compressibility decreases and bulk modulus increases. The elastic properties of magnetic emulsions are modified because a magnetic field tries to orient magnetic moments along the direction of external field axes. That process might be accompanied by a flocculation of droplets. In strong magnetic fields those aggregates may combine into chains oriented along the external magnetic field line.

Also ultrasonic attenuation shows big changes induced by a magnetic field (Figure 10(b)). Under the influence of the field the drops change their shapes and form prolate ellipsoids, the major axis of which is oriented along the field lines. Droplets may also flocculate. That confirms the initial large increase of the attenuation of ultrasonic waves $\Delta \alpha$. The droplets and aggregates are compacted by the magnetic field and have a bigger diameter than the initial droplets, so the friction between the fluid and the aggregate surface provides more attenuation compared with the initial emulsion. In addition, when nonspherical droplets are aligned with acoustic propagation, sound attenuation is higher than 

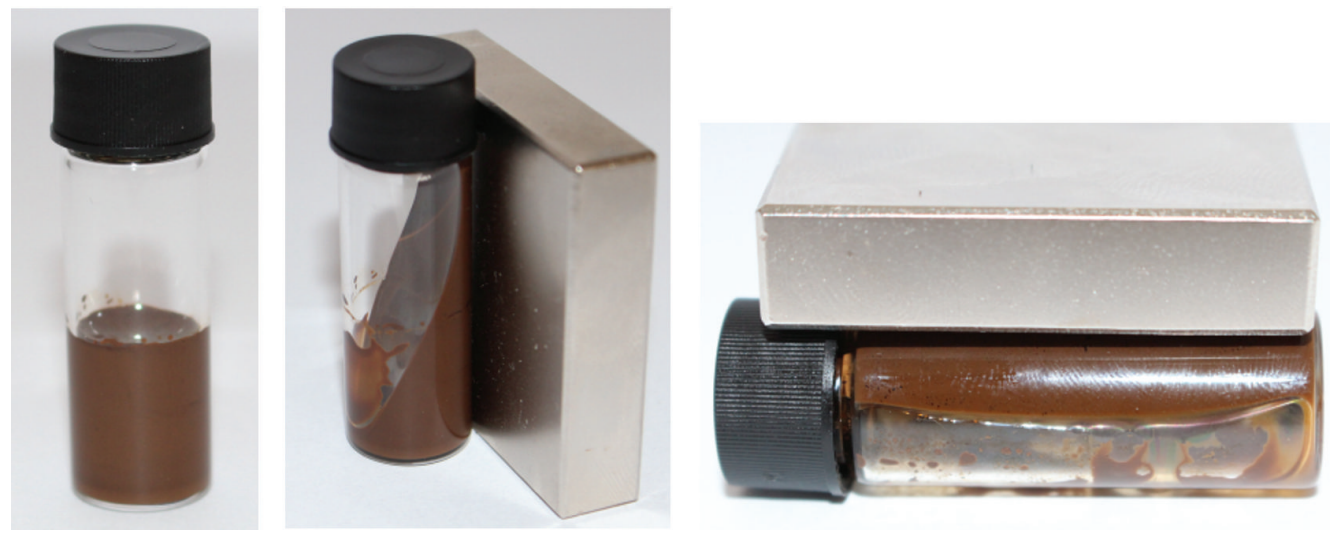

FIGURE 9: The effect of a magnetic field on a magnetite-stabilized emulsion.

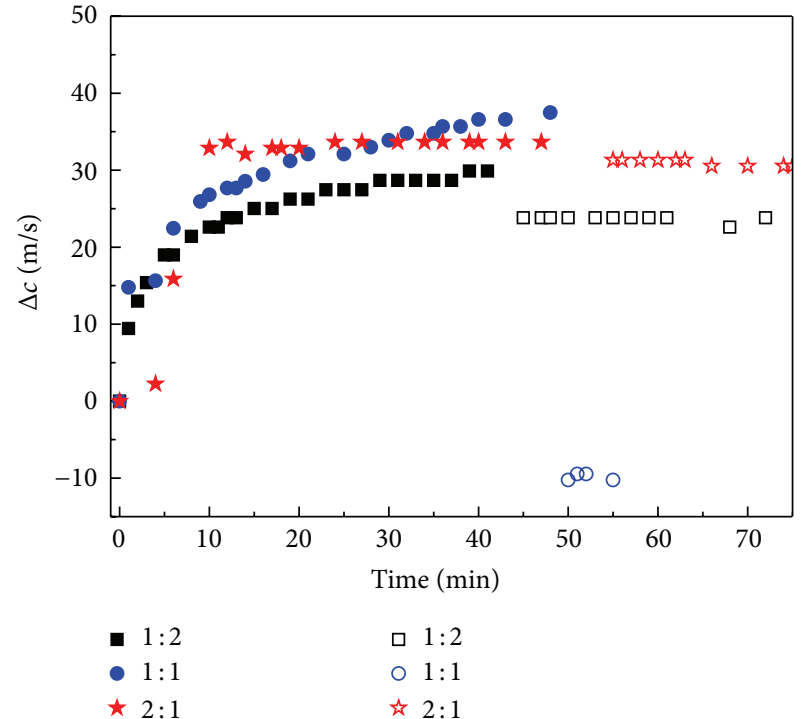

(a)

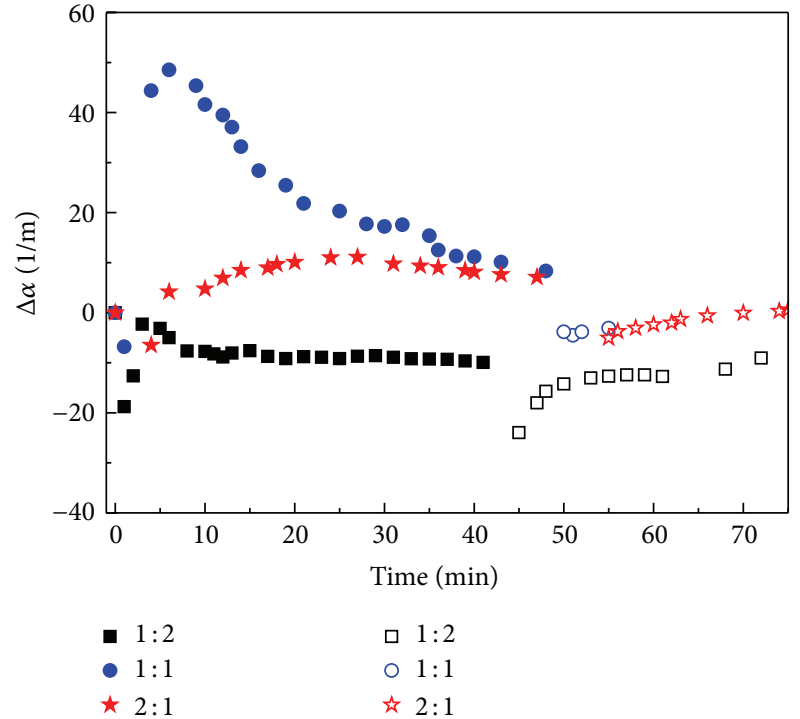

(b)

FIGURE 10: The changes of ultrasonic wave velocity (a) and attenuation (b) as a function of time after the application of a magnetic field. Open symbols represent values after the removal of the magnets.

in the case of a suspension of spherical droplets with the same volume fraction [36]. Then, attenuation decreases, which is caused by the coalescence and the magnetically induced phase condensation. The magnetic colloid separates into coexisting phases. The experimentally observed phase condensation belongs to the most important structuring phenomena in magnetic nanocolloids [37, 38]. The changes are irreversible-after the field has been switched off, acoustic parameters do not return to the initial values.

After 24 hours the magnetic field has been able to totally destabilize the emulsion. The droplets begin to coalesce and the emulsions destabilize to a fully phase-separated system due to the strong attractions and orientation of magnetic particles in the magnetic field direction [11], as indicated in Figure 11 (the delamination of the emulsion into a water and oil suspension of nanoparticles is clearly visible). The magnetically responsive Pickering emulsion is easy to manipulate, and the demulsification is a result of the force applied by the particles [15]. For example, small-strength magnetic fields

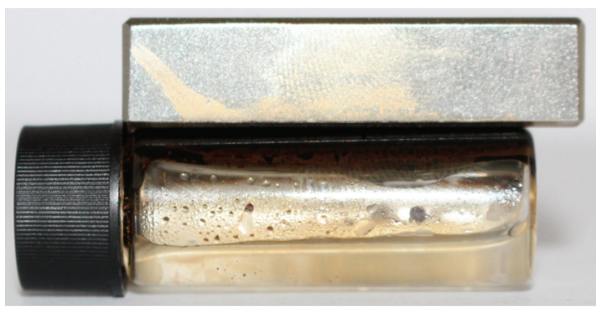

FIGURE 11: A fully phase-separated system under the influence of an external magnetic field.

present the possibility of making the emulsion drops flow in a continuous phase [39].

\section{Conclusion}

The stable magnetic nanoparticle stabilized emulsions $(\mathrm{o} / \mathrm{w})$ were obtained using high-energy ultrasound waves and 
a cavitation mechanism. That is a simple and fast method for the preparation of emulsions. The stability in time of the studied emulsions was confirmed by the monitoring of ultrasonic waves propagation with very low intensity. Measurements of acoustic parameters provided accurate information about the properties of magnetic Pickering emulsions. This study shows that even if macroscopic changes were not observed (no phase separation, sedimentation, or creaming), different processes can take place in the microscale, for example, coagulation which can be investigated by means of acoustic waves. In particular, the measurements of ultrasonic attenuation indicate high stability of emulsions stabilized by magnetite particles as a function of time-a very slow coalescence. The measurements of viscoelastic properties also indicate high stability of the magnetic emulsions prepared with the use of ultrasound method.

It is possible to control Pickering emulsion stability using external stimuli-a magnetic field. When a magnetic field is applied, the emulsion exhibits complex structures. After the introduction of a magnetic field in stable emulsions, droplet coalescence is initiated. Magnetic dipoles interact, changing the structure of the colloids and causing a structural transition. After long time a magnetic field totally destabilizes the emulsion to a fully macrophase-separated system.

\section{Conflict of Interests}

The authors declare that there is no conflict of interests regarding the publication of this paper.

\section{Acknowledgments}

This work was supported by the Polish National Science Centre Grant DEC-2011/03/B/ST7/00194. The authors wish to thank Professor M. Timko from the Slovak Academy of Sciences (Košice) for the samples of the nanoparticle suspensions and Professor P. Czarnecki for providing the optical microscope.

\section{References}

[1] L. L. Schramm, Emulsions, Foams, and Suspensions: Fundamentals and Applications, Wiley, Weinheim, Germany, 2005.

[2] P. Kippax and D. Higgs, "Application of ultrasonic spectroscopy for particle size analysis of concentrated systems without dilution," American Laboratory, vol. 33, no. 23, pp. 8-10, 2001.

[3] S. U. Pickering, "CXCVI.-emulsions," Journal of the Chemical Society, Transactions, vol. 91, pp. 2001-2021, 1907.

[4] R. Aveyard, B. P. Binks, and J. H. Clint, "Emulsions stabilised solely by colloidal particles," Advances in Colloid and Interface Science, vol. 100-102, pp. 503-546, 2003.

[5] D. J. French, P. Taylor, J. Fowler, and P. S. Clegg, "Making and breaking bridges in a Pickering emulsion," Journal of Colloid and Interface Science, vol. 441, pp. 30-38, 2015.

[6] P. Dommersnes, Z. Rozynek, A. Mikkelsen et al., "Active structuring of colloidal armour on liquid drops," Nature Communications, vol. 4, article 2066, 2013.

[7] M. M. Rahman, F. Montagne, H. Fessi, and A. Elaissari, "Anisotropic magnetic microparticles from ferrofluid emulsion," Soft Matter, vol. 7, no. 4, pp. 1483-1490, 2011.
[8] K. T. Wu and Y. D. Yao, "Dynamic structure study of $\mathrm{Fe}_{3} \mathrm{O}_{4}$ ferrofluid emulsion in magnetic field," Journal of Magnetism and Magnetic Materials, vol. 201, no. 1-3, pp. 186-190, 1999.

[9] M. Ivey, J. Liu, Y. Zhu, and S. Cutillas, "Magnetic-field-induced structural transitions in a ferrofluid emulsion," Physical Review E, vol. 63, no. 1, Article ID 011403, 11 pages, 2000.

[10] G. A. Flores, J. Liu, M. Mohebi, and N. Jamasbi, "Magnetic-fieldinduced nonequilibrium structures in a ferrofluid emulsion," Physical Review E, vol. 59, no. 1, pp. 751-762, 1999.

[11] X. Qiao, J. Zhou, B. P. Binks, X. Gong, and K. Sun, "Magnetorheological behavior of Pickering emulsions stabilized by surface-modified $\mathrm{Fe}_{3} \mathrm{O}_{4}$ nanoparticles," Colloids and Surfaces A: Physicochemical and Engineering Aspects, vol. 412, pp. 20-28, 2012.

[12] J. Zhou, L. Wang, X. Qiao, B. P. Binks, and K. Sun, "Pickering emulsions stabilized by surface-modified $\mathrm{Fe}_{3} \mathrm{O}_{4}$ nanoparticles," Journal of Colloid and Interface Science, vol. 367, no. 1, pp. 213224, 2012.

[13] Q. Lan, C. Liu, F. Yang, S. Liu, J. Xu, and D. Sun, "Synthesis of bilayer oleic acid-coated $\mathrm{Fe}_{3} \mathrm{O}_{4}$ nanoparticles and their application in pH-responsive Pickering emulsions," Journal of Colloid and Interface Science, vol. 310, no. 1, pp. 260-269, 2007.

[14] J. Zhou, X. Qiao, B. P. Binks et al., "Magnetic pickering emulsions stabilized by $\mathrm{Fe}_{3} \mathrm{O}_{4}$ nanoparticles," Langmuir, vol. 27, no. 7, pp. 3308-3316, 2011.

[15] Z. Lin, D. Yu, and Y. Li, "Study on the magnetic ODSA-in-water Pickering emulsion stabilized by $\mathrm{Fe}_{3} \mathrm{O}_{4}$ nanoparticle," Colloid and Polymer Science, vol. 293, pp. 125-134, 2015.

[16] W. J. Ahn, H. S. Jung, and H. J. Choi, "Pickering emulsion polymerized smart magnetic poly(methyl methacrylate) $/ \mathrm{Fe}_{2} \mathrm{O}_{3}$ composite particles and their stimulus-response," RSC Advances, vol. 5, no. 29, pp. 23094-23100, 2015.

[17] W. Guo, D. Li, J.-A. Zhu et al., "A magnetic nanoparticle stabilized gas containing emulsion for multimodal imaging and triggered drug release," Pharmaceutical Research, vol. 31, no. 6, pp. 1477-1484, 2014.

[18] M. Kaci, S. Meziani, E. Arab-Tehrany, G. Gillet, I. DesjardinsLavisse, and S. Desobry, "Emulsification by high frequency ultrasound using piezoelectric transducer: formation and stability of emulsifier free emulsion," Ultrasonics Sonochemistry, vol. 21, no. 3, pp. 1010-1017, 2014.

[19] A. S. Dukhin and P. J. Goetz, Characterization of Liquids, Nanoand Microparticulates, and Porous Bodies Using Ultrasound, Elsevier, 2010.

[20] M. Rajnak, J. Kurimsky, B. Dolnik et al., "Dielectric response of transformer oil based ferrofluid in low frequency range," Journal of Applied Physics, vol. 114, no. 3, Article ID 034313, 2013.

[21] A. Józefczak, T. Hornowski, and A. Skumiel, “Temperature dependence of particle size distribution in transformer oilbased ferrofluid," International Journal of Thermophysics, vol. 32, no. 4, pp. 795-806, 2011.

[22] T. Hornowski, A. Józefczak, B. Kołodziejczyk, M. Timko, A. Skumiel, and M. Rajnak, "The effect of particle aggregate shape on ultrasonic anisotropy in concentrated magnetic fluids," Journal of Physics D: Applied Physics, vol. 48, no. 17, Article ID 175303, 2015.

[23] A. Józefczak, T. Hornowski, A. Skumiel et al., "Effect of the molecular weight of poly(ethylene glycol) on the properties of biocompatible magnetic fluids," International Journal of Thermophysics, vol. 33, no. 4, pp. 640-652, 2012. 
[24] A. Skumiel, A. Józefczak, K. Heller, T. Hornowski, and K. Wielgusz, "Investigation of ultrasonic emulsifying processes of a linseed oil and water mixture," Archives of Acoustics, vol. 38, no. 3, pp. 297-301, 2013.

[25] F. Qi, J. Wu, G. Sun, F. Nan, T. Ngai, and G. Ma, "Systematic studies of Pickering emulsions stabilized by uniform-sized PLGA particles: preparation and stabilization mechanism," Journal of Materials Chemistry B, vol. 2, no. 43, pp. 7605-7611, 2014.

[26] B. P. Binks, L. Isa, and A. T. Tyowua, "Direct measurement of contact angles of silica particles in relation to double inversion of pickering emulsions," Langmuir, vol. 29, no. 16, pp. 49234927, 2013.

[27] A. Józefczak, M. Molčan, Z. Rozynek et al., "Properties of magnetosome suspension under the influence of magnetic field," Acta Physica Polonica A, vol. 127, no. 2, pp. 629-631, 2015.

[28] F. Alba, G. M. Crawley, J. Fatkin, D. M. J. Higgs, and P. G. Kippax, "Acoustic spectroscopy as a technique for the particle sizing of high concentration colloids, emulsions and suspensions," Colloids and Surfaces A: Physicochemical and Engineering Aspects, vol. 153, no. 1-3, pp. 495-502, 1999.

[29] P. Mougin, D. Wilkinson, K. J. Roberts, R. Jack, and P. Kippax, "Sensitivity of particle sizing by ultrasonic attenuation spectroscopy to material properties," Powder Technology, vol. 134, no. 3, pp. 243-248, 2003.

[30] B. P. Binks and S. O. Lumsdon, "Influence of particle wettability on the type and stability of surfactant-free emulsions," Langmuir, vol. 16, no. 23, pp. 8622-8631, 2000.

[31] A. M. Atta, G. A. El-Mahdy, H. A. Al-Lohedan, and A. M. El-Saeed, "Preparation and application of crosslinked poly(sodium acrylate)-coated magnetite nanoparticles as corrosion inhibitors for carbon steel alloy," Molecules, vol. 20, no. 1, pp. 1244-1261, 2015.

[32] Y. J. Kim, Y. D. Liu, Y. Seo, and H. J. Choi, "Pickering-emulsionpolymerized polystyrene/ $\mathrm{Fe}_{2} \mathrm{O}_{3}$ composite particles and their magnetoresponsive characteristics," Langmuir, vol. 29, no. 16, pp. 4959-4965, 2013.

[33] O. Bălău, D. Bica, M. Koneracka, P. Kopčansky, D. Susan-Resiga, and L. Vékás, "Rheological and magnetorheological behaviour of some magnetic fluids on polar and nonpolar carrier liquids," International Journal of Modern Physics B, vol. 16, no. 17-18, pp. 2765-2771, 2002.

[34] J. Tang, P. J. Quinlan, and K. C. Tam, "Stimuli-responsive Pickering emulsions: recent advances and potential applications," Soft Matter, vol. 11, no. 18, pp. 3512-3529, 2015.

[35] A. O. Ivanov and O. B. Kuznetsova, "Nonmonotonic fielddependent magnetic permeability of a paramagnetic ferrofluid emulsion," Physical Review E, vol. 85, no. 4, Article ID 041405, 2012.

[36] J. Rodríguez-López, L. Elvira, P. Resa, and F. Montero de Espinosa, "Sound attenuation in magnetorheological fluids," Journal of Physics D: Applied Physics, vol. 46, no. 6, Article ID 065001, 2013.

[37] V. Socoliuc, D. Bica, and L. Vékás, "Magnetically induced phase condensation with asymptotic critical temperature in an aqueous magnetic colloid," Magnetohydrodynamics, vol. 47, no. 2, pp. 201-206, 2011.

[38] A. Y. Zubarev and L. Y. Iskakova, "Yield stress in thin layers of ferrofluids," Physica A: Statistical Mechanics and Its Applications, vol. 365, no. 2, pp. 265-281, 2006.

[39] S. Melle, M. Lask, and G. G. Fuller, "Pickering emulsions with controllable stability," Langmuir, vol. 21, no. 6, pp. 2158-2162, 2005. 

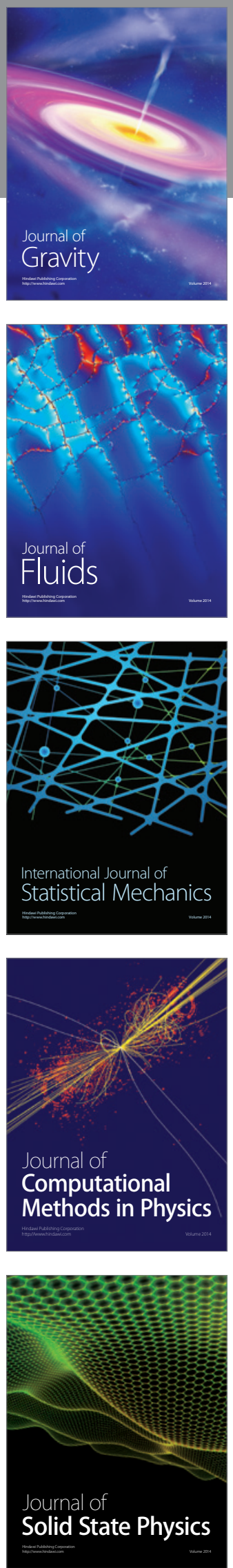

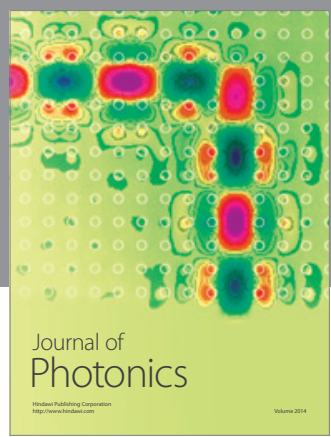

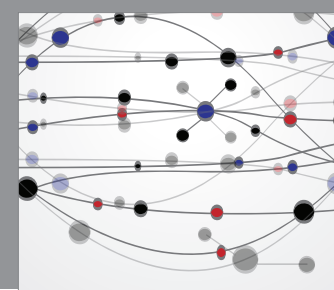

The Scientific World Journal

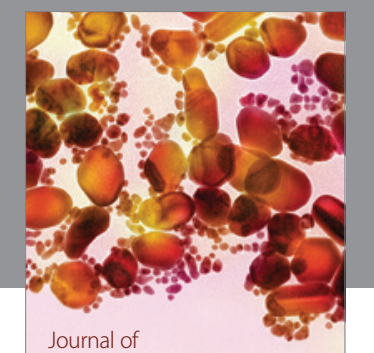

Soft Matter
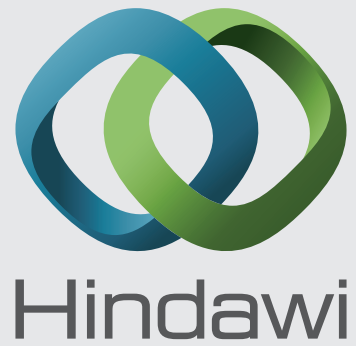

Submit your manuscripts at

http://www.hindawi.com
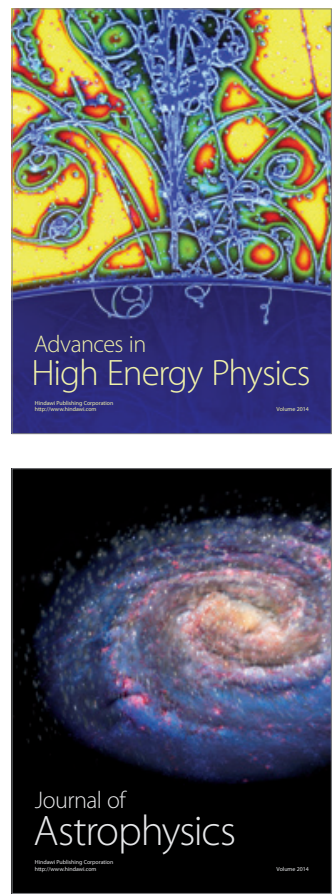
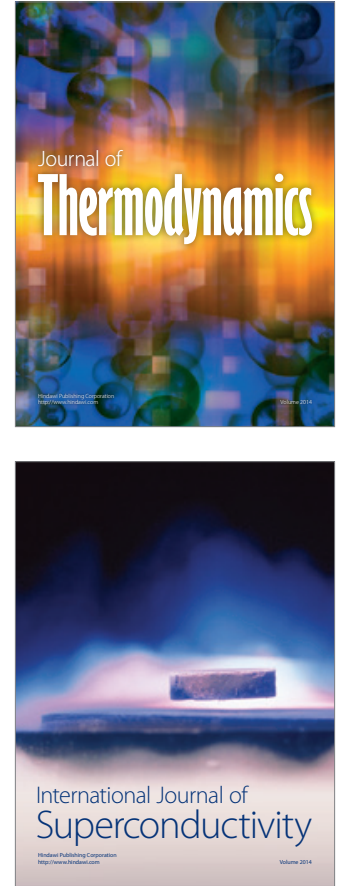
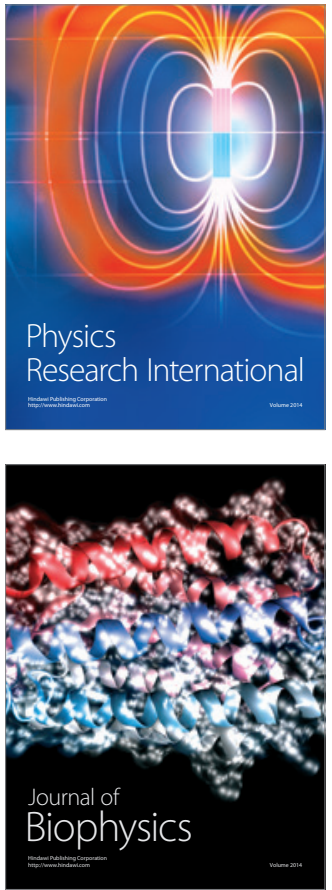
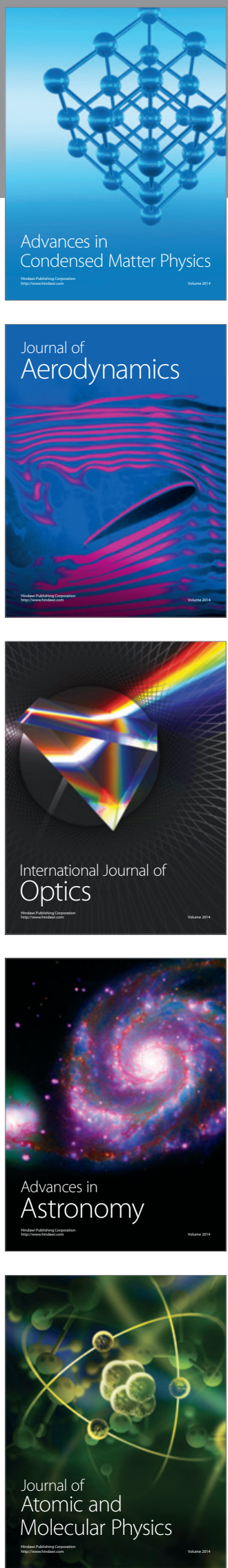